\title{
MICs and minimum fungicidal concentrations of amphotericin B, itraconazole, posaconazole and terbinafine in Sporothrix schenckii
}

\begin{abstract}
Correspondence
Carolina Pereira Silveira carolina.silveira@ufrgs.br
\end{abstract}

Received 3 November 2008 Accepted 7 August 2009

\author{
Carolina Pereira Silveira, ${ }^{1}$ Josep M. Torres-Rodríguez, ${ }^{2,3}$ \\ Eidi Alvarado-Ramírez, ${ }^{2,3}$ Francisca Murciano-Gonzalo, ${ }^{2}$ Maribel Dolande, ${ }^{4}$ \\ Mercedes Panizo ${ }^{4}$ and Vera Reviakina ${ }^{4}$
${ }^{1}$ Centro de Biotecnologia, Universidade Federal do Rio Grande do Sul, PO Box 15005, 91501-970 Porto Alegre, RS, Brazil \\ ${ }^{2}$ Unitat de Recerca en Malalties Infeccioses i Micologia, Institut Municipal d'Investigació Médica, \\ Barcelona, Spain \\ ${ }^{3}$ UDIMAS, Autonomous University of Barcelona, Barcelona, Spain \\ ${ }^{4}$ Mycology Department, Natonal Institute of Hygiene Rafael Rangel, Caracas, Bolivarian Republic of \\ Venezuela
}

The in vitro susceptibility of 62 isolates of Sporothrix schenckii in its mycelial form, from LatinAmerican countries (Peru, Venezuela, Brazil and Uruguay) and Spain, to amphotericin B (AB), itraconazole (IZ), posaconazole (PZ) and terbinafine (TB) was determined by measuring the MICs and minimum fungicidal concentrations (MFCs) using a standardized Clinical and Laboratory Standards Institute method. In general, TB was the most active drug, with the lowest geometric mean (GM) MIC and MFC values amongst isolates from the five countries tested. IZ and PZ showed almost the same activity against all strains tested, except for isolates from Uruguay where IZ gave the highest GM MIC (10.68 $\left.\mathrm{mg} \mathrm{I}^{-1}\right)$. AB showed the widest MIC range (0.03-16.0 $\mathrm{mg} \mathrm{I}^{-1}$ ); however, this drug was less active against $79 \%$ of isolates (MICs above $1 \mathrm{mg} \mathrm{I}^{-1}$ ). MFCs were 5 to 20 times higher than the MICs, but the lowest GM MFC and range values were found for TB. IZ and PZ gave the highest GM MFC. MFC may be a better predictor of therapeutic response than $\mathrm{MIC}$, especially in immunosuppressed patients, making the use of $\mathrm{IZ}$ and $\mathrm{PZ}$ an inappropriate treatment. There were some differences in susceptibility according to the geographical source of the isolates, with the MIC being lower for TB in Venezuelan strains $(P=0.066)$ and the MFC higher for PZ in Peruvian strains $(P=0.02)$. Thus, geographical origin may be important for appropriate treatment, and may relate to the identification of species of the S. schenckii complex.

\section{INTRODUCTION}

Sporothrix schenckii, now considered as a complex (Marimon et al., 2007), is a dimorphic pathogenic fungus that is the aetiological agent of human and animal sporotrichosis. This species is widely distributed in nature and can be found in soil associated with organic plant matter (Ramos-e-Silva et al., 2007). The most frequent clinical manifestation is the subacute or chronic cutaneouslymphatic form, followed by fixed cutaneous infection (Bonifaz et al., 2007). More severe systemic presentations affecting joints, lungs and the central nervous system, as well

Abbreviations: $\mathrm{AB}$, amphotericin $\mathrm{B}$; $\mathrm{GM}$, geometric mean; IZ, itraconazole; MFC, minimum fungicidal concentration; PZ, posaconazole; TB, terbinafine. disseminated infections, have also been described, especially in immunosuppressed patients (Vilela et al., 2007).

Traditional treatment of localized cutaneous-lymphatic sporotrichosis is a saturated solution of potassium iodine given orally for 2-3 months (Kauffman, 1995). The availability of some active systemic antifungals such as ketoconazole, itraconazole (IZ) and terbinafine (TB) allows effective treatment (Chapman et al., 2004) that avoids the secondary effects of iodine. Amphotericin B (AB) has been used for visceral and disseminated forms with varying results (Baker et al., 1989; Kosinski et al., 1992; Kohler et al., 2007). Among the newest antifungals, posaconazole (PZ) seems to be active against $S$. schenckii but limited information on a low number of isolates is available (Scheinfeld, 2007; Marimon et al., 2008). 
The aim of this study was to determine the MICs of $A B, I Z$, $\mathrm{TB}$ and $\mathrm{PZ}$ for clinical strains of $S$. schenckii using the standardized broth microdilution method M38-A recommended by the Clinical and Laboratory Standards Institute (formerly the National Committee on Clinical Laboratory Standards) (NCCLS, 2002). Minimum fungicidal concentrations (MFCs) were also measured to determine the killing power of these antifungals against the mycelial phase of $S$. schenckii.

\section{METHODS}

Strains. In vitro tests were performed on 62 clinical isolates of $S$. schenckii, cultivated from patients with cutaneous or cutaneouslymphatic sporotrichosis from different countries. Strains were isolated in Peru $(n=36)$, Venezuela $(n=15)$, Brazil $(n=6)$, Uruguay $(n=3)$ and Spain $(n=2)$. The isolates were conserved in a skimmed milk suspension at $-20{ }^{\circ} \mathrm{C}$ until use.

Antifungal drugs. According to the manufacturer's instructions, $\mathrm{AB}$ (Sigma-Aldrich), PZ (Schering-Plough), IZ (Janssen Research Foundation) and TB (Novartis) were dissolved in $100 \%$ DMSO, and diluted in RPMI 1640 buffered to $\mathrm{pH} 7.0$ with $0.165 \mathrm{M} \mathrm{3-}(\mathrm{N}$ morpholino)propanesulfonic acid, and supplemented with glucose $\left(18 \mathrm{~g} \mathrm{l}^{-1}\right)$ (Alvarado-Ramírez \& Torres-Rodríguez, 2007). Drug concentrations were $0.03-16.0 \mathrm{mg} \mathrm{l}^{-1}$ for all drugs. Serial dilutions of each drug were placed in microdilution plates and stored at $-30{ }^{\circ} \mathrm{C}$ until use.

Broth microdilution test. Isolates were tested using the microbroth dilution method M38-A (NCCLS, 2002) with slight modification (Alvarado-Ramírez \& Torres-Rodríguez, 2007). Inoculum was obtained by growing the isolates for 5 days at $28 \pm 2{ }^{\circ} \mathrm{C}$ in potato dextrose agar. Aspergillus flavus ATCC 204304 was used for quality control. To prepare the inoculum, conidia were removed from the colony surface with $3-4 \mathrm{ml}$ sterile saline solution by gently scraping the surface. The stock suspension was diluted in saline solution and adjusted spectrophotometrically to an $\mathrm{OD}_{530}$ of $0.05-0.1(80-82 \%$ transmittance), corresponding to $1 \times 10^{5}$ c.f.u. $\mathrm{ml}^{-1}$. The viability of each isolate was confirmed by inoculation to Sabouraud dextrose agar plates and counting the number of c.f.u. $\mathrm{ml}^{-1}$. The MIC end-point criterion was the lowest drug concentration at which there was no visible growth after $72 \mathrm{~h}$ incubation at $28{ }^{\circ} \mathrm{C}$. To obtain the MFC, $10 \mu \mathrm{l}$ of each serial dilution was taken from each well and spread on Sabouraud dextrose agar. Plates were incubated at $28{ }^{\circ} \mathrm{C}$ for $72 \mathrm{~h}$. The MFC was defined as the lowest drug concentration that yielded three or fewer colonies (i.e. $99 \%$ of the inoculum was killed) (EspinelIngroff et al., 2002).

Statistical analysis. The Wilcoxon rank-sum test was used for statistical analysis, with statistical significance being set at $P<0.05$. Results were analysed using the software SPSS v13.0 for Windows (SPSS).

\section{RESULTS AND DISCUSSION}

The geometric means (GMs), range of maximum and minimum values, and 50 and $90 \% \mathrm{MIC} / \mathrm{MFC}$ values were determined. Table 1 shows the MICs and MFCs for the four antifungals for the $62 \mathrm{~S}$. schenkii isolates from the five country group values. MIC values for the reference $A$. flavus strains were within the limits described for $\mathrm{AB}$ and IZ (4 and $0.5 \mathrm{mg} \mathrm{l}^{-1}$, respectively).
In this study, $64 \%$ of $S$. schenkii were susceptible to IZ at an MIC of $0.5 \mathrm{mg} \mathrm{l}^{-1}$. The strains from Uruguay showed the highest GM (10.68 $\left.\mathrm{mg} \mathrm{l}^{-1}\right)$; however, we tested only three isolates and two were resistant. MFCs were higher, with GMs ( $\mathrm{mg} \mathrm{l}^{-1}$ ) of 14.44 (Peru), 11.73 (Venezuela), 14.67 (Brazil), 11.33 (Uruguay) and 16 (Spain). Other authors (McGinnis et al., 2001) have demonstrated good activity of IZ in terms of MIC and MFC values against 100 isolates of S. schenckii. This is in agreement with our MIC results; however, our results showed that IZ had a high MFC for most isolates of $S$. schenckii.

For PZ, MIC values were similar to IZ; the strains from Uruguay showed the highest MFCs, and the strains from Brazil showed a lower GM of $10 \mathrm{mg} \mathrm{l^{-1 }}$. The treatment therapies used in Peru are potassium iodide, ketoconazole, IZ and TB (ongoing clinical trial) (Pappas et al., 2000). There are no data on the susceptibility of Peruvian $S$. schenckii isolates to PZ. TB gave the lowest MIC and MFC values.

As found elsewhere (Alvarado-Ramírez \& TorresRodríguez, 2007; Ellis, 2002), AB showed a wide susceptibility range. The isolates from Peru (GM $1.76 \mathrm{mg} \mathrm{l}^{-1}$ ), Uruguay (GM $1.67 \mathrm{mg} \mathrm{l}^{-1}$ ), Brazil (GM $2.17 \mathrm{mg} \mathrm{l}^{-1}$ ) and Spain (GM $2 \mathrm{mg} \mathrm{l}^{-1}$ ) presented the highest MICs. MFCs were higher than MICs (Table 1). If $\mathrm{AB}$ is the treatment of choice, the MIC of the specific isolate should be determined, as failure of treatment could be associated with the lower susceptibility of $S$. schenckii to this antifungal (McGinnis et al., 2001).

Statistical differences were found according to the geographical source of the isolates for the MIC and MFC, being lower for TB in Venezuelan strains $(P=0.066)$ and higher for PZ in Peruvian strains $(P=0.02)$, respectively.

Neyra et al. (2005) showed no correlation between genotype, geographical origin and clinical form in Peru. Mesa-Arango et al. (2002) also found no association between the virulence of $S$. schenckii and its geographical precedence. Marimon et al. (2007) analysed 127 isolates from different countries, including Brazil, Japan, Peru, Venezuela and the USA, and many proved to be genetically different from each other. Because of this, they proposed three new Sporothrix species, two of which have been associated with human infections (Sporothrix brasiliensis and Sporothrix globosa). The isolates of clinical origin were included in three clades: clade I included only Brazilian isolates and was classified as S. brasiliensis. Clade II included practically all the USA isolates (S. schenckii) and clade III was composed of isolates from China, India, Italy, Japan, Spain and the USA, and were named S. globosa. In that study, phenotypic characteristics were not found to distinguish isolates in clade II, despite this group being genetically heterogeneous. This suggests that our isolates may represent not only $S$. schenckii, but also probably another species such as $S$. brasiliensis, which has the same USA origin as the isolates of Marimon et al. (2007). It will be important to classify these isolates, as they show 
Table 1. MICs, MFCs, range and susceptibilities (\%) at various MICs of four antifungal drugs in strains of S. schenckii, in a mycelial form, from five countries

\begin{tabular}{|c|c|c|c|c|c|c|c|c|c|c|c|c|c|c|c|}
\hline \multirow{2}{*}{$\begin{array}{l}\text { Group }(n) \text { and } \\
\text { antifungal agent }\end{array}$} & \multicolumn{4}{|c|}{$\operatorname{MIC}\left(\mathrm{mg} \mathrm{l}^{-1}\right)$} & \multicolumn{4}{|c|}{$\operatorname{MFC}\left(\mathrm{mg} \mathrm{l}^{-1}\right)$} & \multicolumn{7}{|c|}{ Susceptibility (\%) at MIC (mg $\left.\mathrm{l}^{-1}\right)$ of: } \\
\hline & GM & Range & $50 \%$ & $90 \%$ & GM & Range & $50 \%$ & $90 \%$ & $\leqslant 0.25$ & 0.5 & 1 & 2 & 4 & 8 & $\geqslant 16$ \\
\hline \multicolumn{16}{|c|}{ Group 1 - Peru (36) } \\
\hline $\mathrm{AB}$ & 1.76 & $0.12-16$ & 2 & 2 & 6.03 & $0.5-16$ & 4 & 16 & 5.56 & 22.22 & 19.44 & 50 & - & - & 2.78 \\
\hline $\mathrm{TB}$ & 0.37 & $0.03-1$ & 0.5 & 0.5 & 1.97 & $0.25-8$ & 1 & 4 & 47.22 & 50 & 2.78 & - & - & - & - \\
\hline \multicolumn{16}{|c|}{ Group 2 - Venezuela (15) } \\
\hline $\mathrm{AB}$ & 1.54 & $0.03-2$ & 2 & 2 & 7.57 & $0.5-16$ & 4 & 16 & 6.67 & 13.33 & 13.33 & 66.67 & - & - & - \\
\hline $\mathrm{TB}$ & 0.27 & $0.03-0.5$ & 0.25 & 0.5 & 1 & $0.5-2.0$ & 1 & 2 & 73.33 & 26.67 & - & - & - & - & - \\
\hline $\mathrm{IZ}$ & 0.6 & $0.03-1$ & 0.5 & 1 & 11.73 & $2.0-16$ & 16 & 16 & 6.67 & 66.67 & 26.67 & - & - & - & - \\
\hline $\mathrm{PZ}$ & 0.67 & $0.03-1$ & 0.5 & 1 & 12.13 & $2.0-16$ & 16 & 16 & 20 & 33.33 & 46.67 & - & - & - & - \\
\hline \multicolumn{16}{|c|}{ Group 3 - Brazil (6) } \\
\hline $\mathrm{PZ}$ & 0.58 & $0.25-1$ & 0.5 & 1 & 10 & $2.0-16$ & 8 & 16 & 33.33 & 33.33 & 33.33 & - & - & - & - \\
\hline \multicolumn{16}{|c|}{ Group 4 - Uruguay (3) } \\
\hline $\mathrm{AB}$ & 1.67 & $1.0-2.0$ & 2 & 2 & 6.67 & $2.0-16$ & 2 & 16 & - & - & 33.33 & 66.67 & - & - & - \\
\hline $\mathrm{TB}$ & 0.19 & $0.06-0.25$ & 0.25 & 0.25 & 1.04 & $0.12-2$ & 1 & 2 & 100 & - & - & - & - & - & - \\
\hline $\mathrm{IZ}$ & 10.68 & $0.03-16$ & 16 & 16 & 11.33 & $2.0-16$ & 16 & 16 & 33.33 & - & - & - & - & - & 66.67 \\
\hline $\mathrm{PZ}$ & 1.35 & $0.06-0.25$ & 0.25 & 0.25 & 11 & $1.0-16$ & 16 & 16 & 100 & - & - & - & - & - & - \\
\hline \multicolumn{16}{|c|}{ Group 5 - Spain (2) } \\
\hline $\mathrm{AB}$ & 2 & 2 & 2 & 2 & 6 & $4.0-8.0$ & 4 & 8 & - & - & - & 100 & - & - & - \\
\hline $\mathrm{TB}$ & 0.5 & 0.5 & 0.5 & 0.5 & 1.5 & $1.0-2.0$ & 1 & 2 & - & 100 & - & - & - & - & - \\
\hline $\mathrm{IZ}$ & 1.5 & $1.0-2.0$ & 1 & 2 & 16 & 16 & 16 & 16 & - & - & 50 & 50 & - & - & - \\
\hline $\mathrm{PZ}$ & 1.5 & $1.0-2.0$ & 1 & 2 & 16 & 16 & 16 & 16 & - & - & 50 & 50 & - & - & - \\
\hline
\end{tabular}

different responses to antifungal agents that could be critical for appropriate patient management.

Recently, the susceptibilities of 92 isolates belonging to 5 species of Sporothrix were determined against 12 antifungal agents. TB, ketoconazole and $\mathrm{PZ}$ were shown to be the most active drugs. The authors suggested that $\mathrm{PZ}$ is a better therapeutic agent in the treatment of systemic infections than $\mathrm{AB}$ and IZ, which gave high MICs (Marimon et al., 2008). These isolates in general gave MICs higher than our strains for $\mathrm{IZ}, \mathrm{AB}$ and $\mathrm{PZ}$, indicating that antifungal susceptibilities are strain-dependent. In contrast, for TB, the MICs were very similar to those seen in this study.

Our results suggest that some differences may exist in susceptibility to some antifungals (TB and PZ) according to the geographical origin of the strains. Further studies are needed, with larger numbers of isolates from other countries and molecular typing of these isolates to investigate whether they are genetically different or not and whether different species in the S. schenckii complex are important, to confirm these findings.
The results of this study support the therapeutic use of TB as a first option for the treatment of sporotrichosis (Chapman et al., 2004) and suggest consideration of the MFC values for IZ before starting treatment. PZ could be another alternative treatment, but further clinical studies are necessary before proposing this drug as a therapeutic option.

\section{ACKNOWLEDGEMENTS}

We would like to thank Dr Sydney Hartz Alves from Brazil and Dr Beatriz Bustamante from Peru for generously providing some of the isolates used in this study.

\section{REFERENCES}

Alvarado-Ramírez, E. \& Torres-Rodríguez, J. M. (2007). In vitro susceptibility of Sporothrix schenckii to six antifungal agents determined using three different methods. Antimicrob Agents Chemother 51, 2420-2423. 
Baker, J. H., Goodpasture, H. C., Kuhns, H. R., Jr \& Rinaldi, M. G. (1989). Fungemia caused by an amphotericin B-resistant isolate of Sporothrix schenckii. Successful treatment with itraconazole. Arch Pathol Lab Med 113, 1279-1281.

Bonifaz, A., Saúl, A., Paredes-Solis, V., Fierro, L., Rosales, A., Palacios, C. \& Araiza, J. (2007). Sporotrichosis in childhood: clinical and therapeutic experience in 25 patients. Pediatr Dermatol 24, 369372.

Chapman, S. W., Pappas, P., Kauffman, C., Smith, E. B., Dietze, R., Tiraboschi-Foss, N., Restrepo, A., Bustamante, A. B., Opper, C. \& other authors (2004). Comparative evaluation of the efficacy and safety of two doses of terbinafine ( 500 and $1000 \mathrm{mg} \mathrm{day}^{-1}$ ) in the treatment of cutaneous or lymphocutaneous sporotrichosis. Mycoses 47, 62-68.

Ellis, D. (2002). Amphotericin B: spectrum and resistance. J Antimicrob Chemother 49, 7-10.

Espinel-Ingroff, A., Fothergill, A., Meter, J., Rinaldi, M. G. \& Walsh, T. J. (2002). Testing conditions for determination of minimum fungicidal concentrations to new and established antifungal agents for Aspergillus spp.: NCCLS collaborative study. J Clin Microbiol 40, 3204-3208.

Kauffman, C. A. (1995). Old and new therapies for sporotrichosis. Clin Infect Dis 21, 981-985.

Kohler, L. M., Hamdan, J. S. \& Ferrari, T. C. (2007). Successful treatment of a disseminated Sporothrix schenckii infection and in vitro analysis for antifungal susceptibility testing. Diagn Microbiol Infect Dis 58, 117-120.

Kosinski, R. M., Axelrod, P., Rex, J. H., Burday, M., Sivaprasad, R. \& Wreiole, A. (1992). Sporothrix schenckii fungemia without disseminated sporotrichosis. J Clin Microbiol 30, 501-503.

Marimon, R., Cano, J., Gené, J., Sutton, D. A., Kawasaki, M. \& Guarro, J. (2007). Sporothrix brasiliensis, S. globosa, and S. mexicana, three new Sporothrix species of clinical interest. J Clin Microbiol 45, 31983206.

Marimon, R., Serena, C., Gené, J., Cano, J. \& Guarro, J. (2008). In vitro susceptibilities of five species of Sporothrix. Antimicrob Agents Chemother 52, 732-734.

McGinnis, M. R., Nordoff, N., Li, R. K., Pasarell, L. \& Warnock, D. W. (2001). Sporothrix schenckii sensitivity to voriconazole, itraconazole and amphotericin B. Med Mycol 39, 369-371.

Mesa-Arango, A. C., Reyes-Montes, M. R., Pérez-Mejía, A., NavarroBarranco, H., Souza, V., Zúñiga, G. \& Toriello, C. (2002). Phenotyping and genotyping of Sporothrix schenckii isolates according to geographic origin and clinical form of sporotrichosis. J Clin Microbiol 40, 3004-3011.

NCCLS (2002). Reference Methods for Broth Dilution Antifungal Susceptibility Testing of Filamentous Fungi, approved guideline M38A. Wayne, PA: National Committee for Clinical Laboratory Standards.

Neyra, E., Fonteyne, P. A., Swinne, D., Fauche, F., Bustamante, B. \& Nolard, N. (2005). Epidemiology of human sporotrichosis investigated by amplified fragment length polymorphism. J Clin Microbiol 43, 1348-1352.

Pappas, P. G., Tellez, I., Deep, A. E., Nolasco, D., Holgado, W. \& Bustamante, B. (2000). Sporotrichosis in Peru: description of an area of hyperendemicity. Clin Infect Dis 30, 65-70.

Ramos-e-Silva, M., Vasconcelos, C., Carneiro, S. \& Cestari, T. (2007). Sporotrichosis. Clin Dermatol 25, 181-187.

Scheinfeld, N. (2007). A review of the new antifungals: posaconazole, micafungin, and anidulafungin. J Drugs Dermatol 6, 1249 1251.

Vilela, R., Souza, G. F., Fernandez Cota, G. \& Mendoza, L. (2007). Cutaneous and meningeal sporotrichosis in a HIV patient. Rev Iberoam Micol 24, 161-163. 